\title{
Localised FM Digital Audio Broadcasting using WiFi Mesh Networks
}

This paper was downloaded from TechRxiv (https://www.techrxiv.org).

\section{LICENSE}

CC BY 4.0

SUBMISSION DATE / POSTED DATE

$13-12-2020$ / 21-09-2021

CITATION

Verma, Ashvin; Kar, Somesh; Ahuja, Priyanshi (2020): Localised FM Digital Audio Broadcasting using WiFi Mesh Networks. TechRxiv. Preprint. https://doi.org/10.36227/techrxiv.13371068.v3

$\mathrm{DOI}$

10.36227/techrxiv.13371068.v3 


\section{Localised FM Digital Audio Broadcasting using WiFi Mesh Networks}

\author{
Ashvin Verma \\ Delhi Public School, R.K Puram \\ New Delhi, India 110022 \\ Email: ashvin.vermarocks@gmail.com
}

\author{
Somesh Kar \\ Delhi Public School, R.K Puram \\ New Delhi, India 110022 \\ Email: somesh.kar@gmail.com
}

\author{
Priyanshi Ahuja \\ Delhi Public School, R.K Puram \\ New Delhi, India 110022 \\ Email: priyanshi.ahuja09@gmail.com
}

\begin{abstract}
Digital Audio Broadcasting via FM radio continues to be popular, even today, owing to its accessibility, especially in rural areas where even common feature phones and affordable radio receivers are able to freely receive both, mainstream and local vernacular transmissions without any subscription charge. Additionally, it is established that broadcasts undertaken at a local level can play an instrumental role in facilitating cultural expression and aiding information delivery at a local level However, local broadcasts via the current Community Radio Stations (CRS) are not only expensive but also unsuitable for remote, isolated regions with their rugged terrains.

The authors hereby propose a novel approach to help address these current problems through a distributed system for Digital Audio Broadcasting. Multiple FM transmitting nodes (using VHF II: 87.5-100 MHz) form a WiFi Mesh Network to receive digital audio signals to broadcast from 1 hub node. The components used are off-the-shelf, cost-effective, and modular. It is more costeffective than current CRS systems for mid-scale local broadcasts, with essentially no data loss between the transmitter units in deployment. Additionally, unlike relays, an existing alternative, built-in redundancy in the system ensures reliability, and caching protects against minor network disturbances when recordings are being broadcast. This approach allows for flexible and efficient deployments with wide, precise and accurate coverage, notably remaining equally effective in areas with an uneven terrain, thereby expanding its potential applications.

Experiments involving the system were conducted by varying the audio samples and the conditions, for which the evaluation metrics included the PSNR, PSD spectrograms, DFTs, signal strength, and data transmission rate to gauge the transmission quality, range and scalability. Results included a moderate data rate of $12 \mathrm{mbits} / \mathrm{s}$ at a 100 metres separation with obstacles from the transmitter, which is sufficient for the broadcasting and fast caching of high-fidelity audio.
\end{abstract}

Keywords-WiFi mesh network, signal processing, local broadcasting, rural technology, FM transmissions, single board computer (SBC), commercial off the shelf (COTS).

\section{INTRODUCTION}

Radio transmission involves overcoming major hurdles including Radio-Frequency Interference (RFI) and fading caused by signals reaching from multiple paths, and in the case of Line of Sight propagation, obstruction by obstacles is another notable consideration, therefore these factors have been the focal point. Analysing Community Radio Stations, they are expensive (setup cost of 1.5 million Indian Rupees(INR) [1]), scale poorly in uneven terrain and are power-intensive[2].

In response, the authors propose a novel approach using a system of Frequency Modulation (FM) transmitters connected via a WiFi Mesh Network to transmit an audio stream from the hub node to every other node for broadcast. This network can be scaled and configured according to the requirements due tof its usage of Commercial Off the Shelf (COTS) components and open-source software in order to accommodate requirements such as connecting to the internet, ensuring signals traverse uneven terrain and other additional capabilities. It requires only a line of sight between transmitters, with WiFi ensuring fullfidelity transmission, and the presence of a cache preventing issues due to minor network disruptions for recordings.

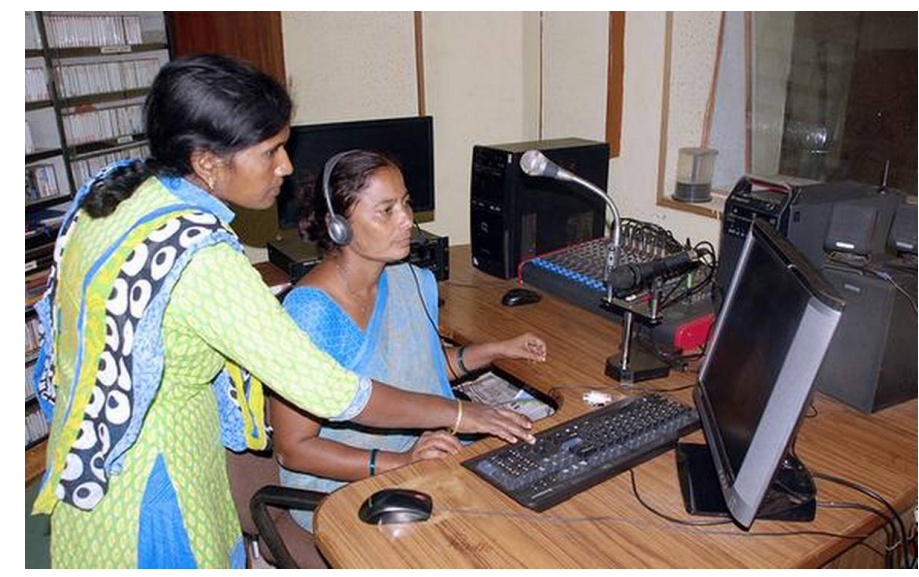

Fig. 1: Traditional Rural Community Radio Broadcast Station Source: https://timesofindia.indiatimes.com/

Section 1 introduces the problem, examining social literature and offers an overview of the solution. Section 2 discusses the methodology of the setup, system configurations. Section 3 introduces the setup of the experiments, whose data is presented via Peak Signal to Noise Ratio (PSNR), Discrete Fourier Transform (DFT), and Power Spectral Density (PSD) spectrogram output and interpreted. Section 4 concludes the paper by summarising the key points and results from the study. Appendix B covers the costing of components.

\section{A. Literature Review}

FM transmission systems suffer from the limitation of requiring a direct line of sight, which may be troublesome in hilly regions. Current distributed systems which address this issue for FM transmission primarily use relays[3]; however, 
these simply rebroadcast the degraded signal they receive, becoming corrupted through accumulating transmission errors. Rural WLAN Mesh Networks on the other hand, are currently used solely for delivering broadband internet access, which still remains inaccessible despite gradual increases in availability. After examining other current methods of information broadcasting globally, it is apparent that their ubiquity varies drastically. Below, the rural areas of two regions of interest with significant socio-cultural, economic, and geographical differences are discussed for analysis: India and a generalised overview across countries in the African continent.

India: 5 widespread means of information dissemination are considered: print, television, national radio, cellular networks, broadband internet. Traditional means (national and state television and radio) remain ubiquitous, however, they cannot fulfill local broadcasting needs. On the other hand, while cellular and broadband networks are increasingly available, they are not universally accessible (due to economical barriers) as they require modern equipment[4]. Literacy in a language that the device supports is also an implicit requirement in a country where there are 22 major recognized languages[5], and 1369 rationalized mother tongues[6] with a literacy rate of $64.8 \%$ [6] .

Some mediums fulfilling the need for localized, decentralized information dissemination are existing community radio stations and local print. The print medium is a traditional source of information but its consumption is limited to the literate. Further, it is also slower in information delivery, and is unusable for urgent broadcasts. Discussing the last medium in India, according to a report on community radio stations by the MIB, Government of India [7], they have significant impact on local cultural and personal expression, among other effects, as benefactors can easily receive transmissions using affordable receivers or accessible feature phones, which require no subscription to the broadcast. Additionally, anywhere from 7,000 to 154,000 households per state were under coverage. Note, however, that while the range of each system is 10 $15 \mathrm{~km}$, smaller villages and communities may require only smaller deployments and can benefit from more affordable deployments, given the expensive and immobile equipment current solutions require (costing 1.5 million INR) [1].

African Countries: Literacy rates in African countries are at an average $70 \%$, lagging drastically behind the world literacy rate average of $90 \%$ [8]. The region features between 12502100 natively spoken languages [9], with some countries also having multiple official languages. Despite intentions to increase the coverage of mediums, especially television and mobile networks, their operation requires a constant source of electricity and therefore access continues to remain limited, as most of the population lives in rural areas with very low population density, making it hard to justify the infrastructure investment required. Additionally, since these services are uniformly priced for consumers, average revenue is optimised over a greater number of users by cellular and internet service providers, causing these services to remain prohibitively expensive for some [4].
Here, community radio can then allow many to keep up with the developments in their local area, useful broadcasts including agricultural advisories, as well as other news delivered by local authorities. The radio's penetration factor is approximately 1 per 5 people, whereas the penetration rate for telephone is far lower at 1 per 100 people. There are often radio "talk clubs" where people come together to listen to the radio and have discussions [10]. Radios are especially crucial for countries with higher illiteracy rates and many vernacular speakers, where the oral nature of the medium makes it even more accessible and beneficial. Community radio stations have had significant success in the African continent in giving a voice to the poor, with further room for improvement [11] [2].

There is a need expressed by governments and the United Nations for expanding the reach of community radio. The authors further conclude that there is currently a lack of affordable and easily scalable modular community radio broadcast methods, especially those which work well across hilly terrain and are capable of accessing information channels such as the internet and aim to provide a functional method to mediate this shortfall.

\section{Methodology}

\section{A. Overview}

The proposed approach consists of a system of transmitter units connected via a WLAN mesh network by the $802.11 \mathrm{~s}$ standard [12] spread across a coverage area. Each transmitter unit, along with its connected accessories, is termed as a "node" of the network. The hub node has a microphone and performs preliminary audio processing before the WiFi Mesh Network propagates the audio stream across multiple children nodes.

Each node, including the hub node then processes the received audio stream to transmit as an FM signal in the VHF Band II (87.5-100 MHz), which consumer radio receivers are configured to receive from as it is commonly used for Digital Audio Broadcasting [13]. Each node utilizes low levels of power and can be placed strategically to provide required the network coverage.

\section{B. Single Node Setup}

For their setup, the authors have used the Raspberry Pi Zero $\mathrm{WH}^{\mathrm{TM}}$ (with pre-soldered headers) for every node, a lowcost COTS Single Board Computer (SBC) with support for $802.11 \mathrm{n} / \mathrm{s}$ wireless LAN, a $1 \mathrm{GHz}$ single-core $\mathrm{CPU}, 512 \mathrm{MB}$ RAM, and mini-HDMI (for a screen) and USB OTG ports. It is powered via micro-USB power[14]. For generating and transmitting signals, multiple approaches were tested.

The functioning of the radio transmission from any node is based on the Python library PiFmAdv [15] calling a C program that maps the Peripheral Bus in physical memory into the virtual address space using / $\mathrm{dev} / \mathrm{mem}$ and mmap with root access. Next, it enables the clock generator module and sets it to output on GPIO pin 4 at $16 \mathrm{~mA} / 3.3 \mathrm{~V}$. It sets a particular carrier frequency, which FM radios detect and stop the white noise. This is when the required audio is transmitted in the form of a WAV file. 


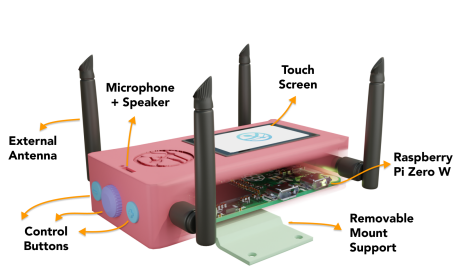

(a) Conceptual Render

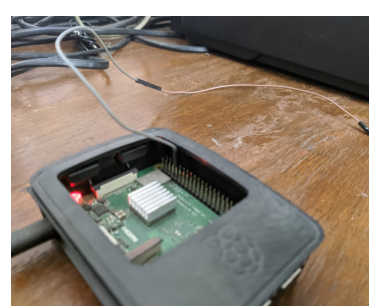

(b) Raspberry Pi Zero WH Setup
Fig. 2: Depiction of Node

Initially, modulation was attempted using the fractional divider, which makes the audio signal using the CPU clock cycles on the GPIO pins of the Raspberry Pi Zero WH; FM broadcasts were broadcast with a piece of ordinary wire connected to the GPIO pin 4. However, signal quality was poor and had significant noise.

The Phase-Locked Loop Clock in the Raspberry Pi's GPU was a better alternative in testing, which, in conjunction with a piece of wire acting as a low-cost antenna, functions as a capable FM transmitter by modulating an AC current to match a reference frequency, for generating a signal resonating at the desired FM frequency. For further research, the usage of external and directional antennas for FM and WiFi could significantly enhance the system's range and strength. All the $\mathrm{DAB}$ antennas used in the experiment are omnidirectional to maximise local coverage by nodes.

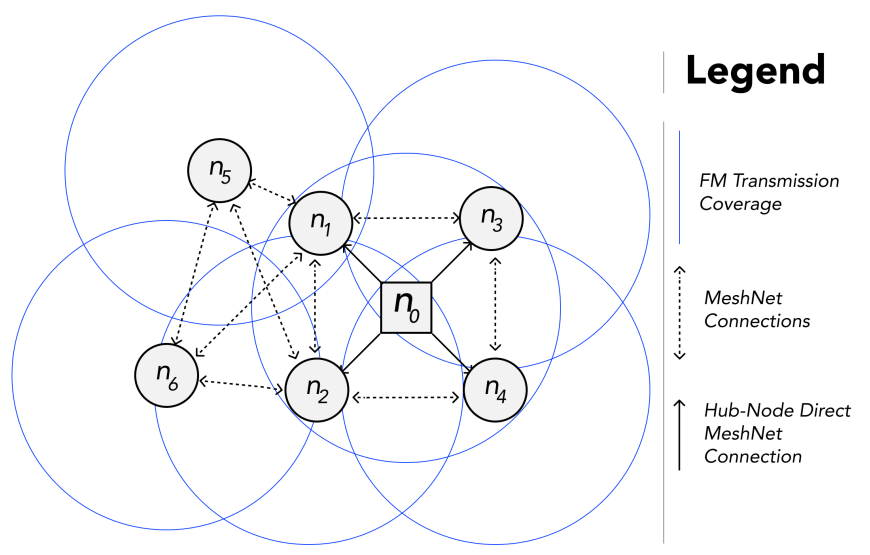

Fig. 3: Representation of WiFi Mesh Network Topology with FM Transmission

\section{Network Configuration}

The WLAN Mesh Network uses the batman-adv wrapper. For recordings, starting at the hub $n_{0}$, the WAV file propagates to every node in the network. The first broadcast node has a depth of 1 , and for each hop of the stream, the child node gets a depth 1 higher than the parent. A mesh network allows for flexible deployment since only LoS between directly connected nodes is required, allowing the network to be deployed selectively or over uneven terrain and also overcome the effects of multipath fading, which is remedied by ACK acknowlegement signal verification to ensure full fidelity. Additionally, the MeshNet allows the presence of redundant nodes in the network, whose failure does not cause the entire network or large regions of it to undergo issues. Caching of recorded broadcasts is achieved through downloading the audio stream at a bit rate far faster than real-time broadcasts require.

Since destructive interference may be caused if nearby units' transmissions are out of phase, directional antennae may be considered for future research to minimise overlap in broadcast regions. Each node may be equipped with a solar panel and be self-sufficient, requiring minimal clearing of the solar panel surface routinely and so it may be kept at a higher location for better Line of Sight transmission. For the experiment setup, the authors have used the Portronics POR-141 to receive FM signals, routing the output via an AUX cable to a Zoom H1 portable recorder.

\section{RESULTS \& DisCUSSION}

Cloud-hosted interactive Python notebooks were used for analysis, available at http://ashverma.co/colabresearch. The Waveform Audio File Format (WAV/WAVE) was used for transmission and reception, as it is uncompressed, for optimal fidelity. For transmission, a carrier frequency of $99.5 \mathrm{MHz}$ is used. A DFT and Waveform have been obtained from each audio clip, along with PSD Spectrograms through GNU Octave, for which the Blackman window [16] is used to minimize discontinuities and power leakage.

For PSNR calculations (in $\mathrm{dB}$ ) for 2 digital signals, where $\mathrm{A}, \mathrm{B}$ are the signals to be compared, each with $\mathrm{k}$ elements and MAX $_{\mathrm{I}}$ as maximum magnitude (for data type):

$$
\operatorname{MSE}(\mathrm{A}, \mathrm{B})=\frac{\sum_{i=1}^{k}\left(A_{i}-B_{i}\right)^{2}}{k}, \mathrm{PSNR}=10 \cdot \log _{10} \frac{\mathrm{MAX}_{I}^{2}}{\mathrm{MSE}} .
$$

Figure 4 shows and labels the locations P1, P2, P3 and P4 used in Experiments 1-4. The walls were 0.3 meters in thickness and made of concrete. The signals were cropped out from the recording window to account for human error.

\section{A. Experiment 1}

The audio file used here was of a monologue by a single speaker transmitted and received as a WAV file at a sampling rate $F_{s}=44100 \mathrm{~Hz}$ and duration $T=64.85 \mathrm{~s}$, represented in Figure 5. The same audio file is used in both parts of Experiment 1. The codec used in the transmission is PCM S16 LE (Little Endian), with 16 bits/sample. A window size of 1024 samples was used for PSD spectrograms, as frequency resolution was preferred over temporal resolution to clearly analyse the profile of background noise.

1) Hub Node FM Transmission: Experiment 1(a) was conducted to determine the range of the DAB signal from a node with obstructions in-between. The "hub" node transmitted from point $\mathrm{P} 1$, with the receiver in the nearby room at point P2 at a distance of 6 metres. The codec used in reception is 


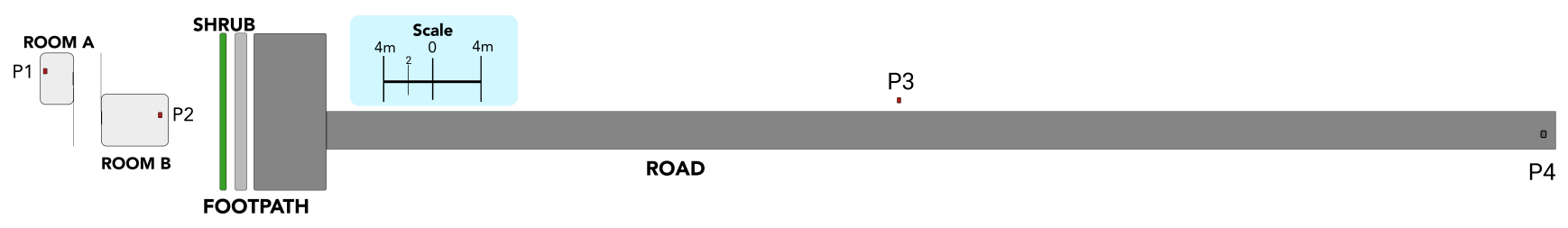

Fig. 4: Overhead Schematic for Setup of Experiments (distances to scale)

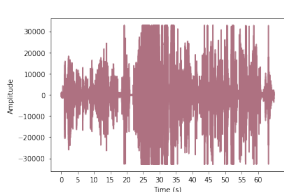

(a) Waveform

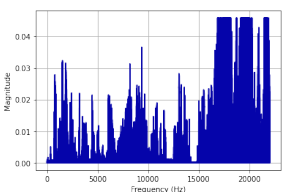

(b) Discrete Fourier Transform

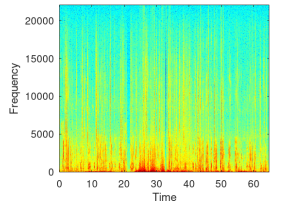

(c) PSD Spectrogram
Fig. 5: Representative Plots for Source Signal from Experiment 1

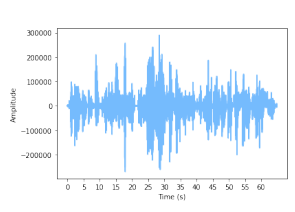

(a) Waveform

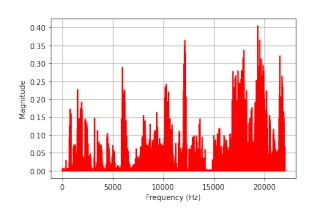
(b) Discrete Fourier Trans-
form

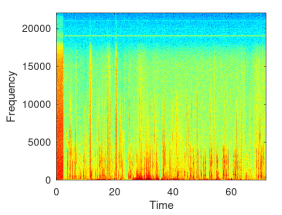

(c) PSD Spectrogram
Fig. 6: Representative Plots for Received Corrupted Signal from Experiment 1(a)

PCM S24 LE (Little Endian), with 24 bits/sample, which were scaled down to 16 bits/sample for PSNR.

The corrupted audio clip had distortions, and the signal strength seems to have weakened. Comparing the DFT distributions, there appear to be clusters of distortion around $10 \mathrm{kHz}$, above the vocal range of human voice, and so a low-pass filter may be considered to increase clarity if musical instruments are not in play. The PSD spectrogram broadly correlates. A PSNR of $68.45 \mathrm{~dB}$ was measured using Eq. 2 .

2) Child Node Transmission: The received transmission was recorded in PCM S16 LE. This test was conducted to test the effect of increasing distance and observing conservation of fidelity in transmitting via a MeshNet. The hub node was present at point $\mathrm{P} 1$, and the child node at $\mathrm{P} 2$ as per Figure 4 and both were transmitting concurrently. The receiver was at point P3, and the distance between the receiver and the child node was 30 metres.

There were some distortions across the frequency spectrum, according to the PSD spectrogram and the TFR, which seems to be due to a combination of constructive and destructive interference, causing amplitude spikes in the signal and shrill distortions in audio perception. A PSNR of $63.92 \mathrm{~dB}$ was measured.

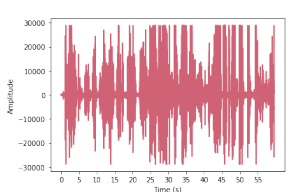

(a) Waveform

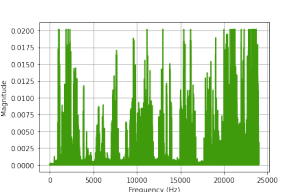

(b) Discrete Fourier Trans form

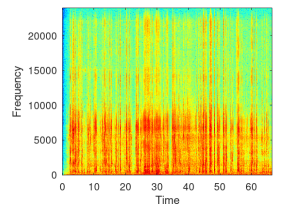

(c) PSD Spectrogram
Fig. 7: Representative Plots for Received Corrupted Signal from Experiment 1(b)

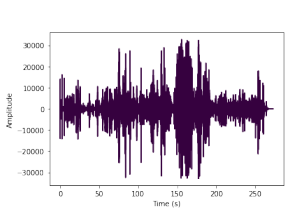

(a) Waveform

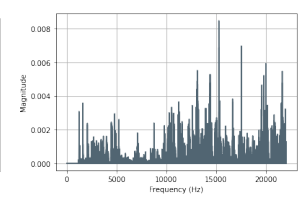

(b) Discrete Fourier Transform

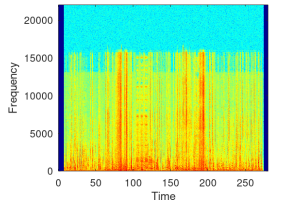

(c) PSD Spectrogram
Fig. 8: Representative Plots for Broadcast Signal for Experiment 2

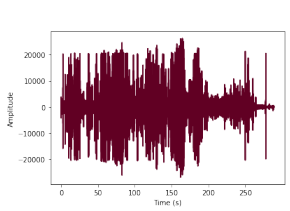

(a) Waveform

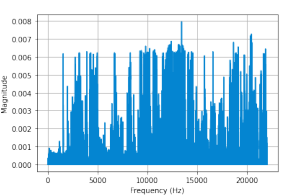

(b) Discrete Fourier Transform

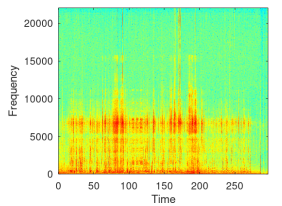

(c) PSD Spectrogram
Fig. 9: Representative Plots for Received Corrupted Signal from Experiment 2

\section{B. Experiment 2: Human Audio received from Depth-3 Nodes}

This test was conducted to further test the performance of the mesh network by transmitting from a depth-3 node (since the transmission has likely hopped twice) across a greater distance and with a more detailed audio of greater duration. The "hub" node at point P1 transmitted the audio via MeshNet, which eventually reached the child node at P4 after a couple of hops, and performed the DAB transmission concurrently. The receiver at $\mathrm{P} 4$ was at a distance of 60 metres. A window size of 4096 samples was used (due to the long duration, setting a shorter window gave minute temporal details).

The audio clip used for this experiment, a conversation 


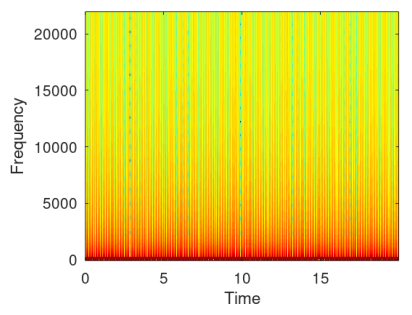

(a) Source Pulse PSD

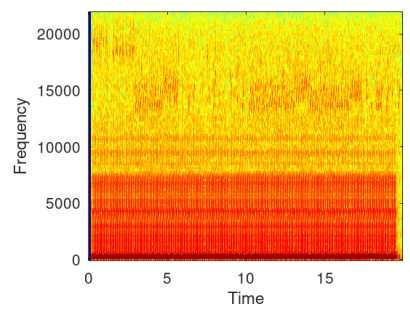

(b) Corrupted Pulse PSD

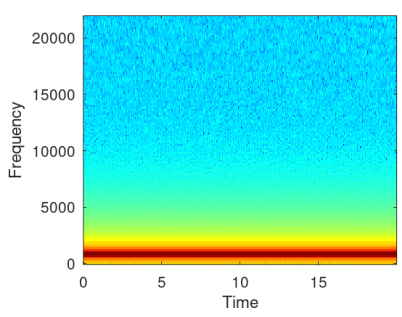

(c) Source A4 PSD

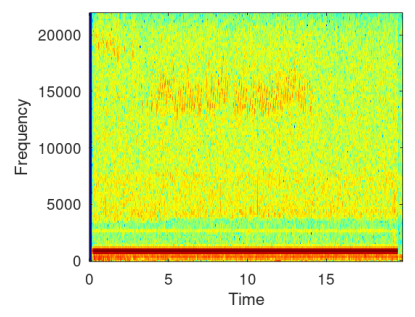

(d) Corrupted A5 PSD

Fig. 10: Representative Plots for Experiment 3: Pulse-wave \& A5 $(880 \mathrm{~Hz})$ Signals

of duration $T=280 \mathrm{~s}$. was a raw WAV at a sampling rate $F_{s}=44100 \mathrm{~Hz}$. The codec used to transmitting and receive was the PCM S16 LE, where S16 specifies 16 bits/sample.

The corrupted audio clip was audible, with a perceivable "crackle", appearing as salt-and-pepper noise on the PSD spectrogram across all frequency bins. A PSNR of $60.38 \mathrm{~dB}$ was measured, lower than earlier experiments due to increased distance and obstacles.

\section{Experiment 3: Sine wave \& Pulses}

A pure-tone sine wave of $880 \mathrm{~Hz}$, the A5 note, was generated to more closely test the distortion in frequency bins, and a $50 \%$ duty-cycle square-wave of frequency $100 \mathrm{~Hz}$ to test distortion in the temporal qualities of the signal. The experiment setup was identical to Experiment 1(b).

A smaller window of 256 samples was chosen for the PSD spectrogram, as temporal resolution was of priority. In Figure 10, in the subfigures representing the square-wave pulses, the corruption seems to predominantly be due to an overlap of the signal with background noise playing a minor role. In the subfigures representing the A5 note signal, the noise is limited to beyond $3.5 \mathrm{kHz}$, but clusters around $15 \mathrm{kHz}$ with limited overlap of signal and moderate levels of background noise are present.

\section{Network Considerations}

The WiFi performance of the Raspberry Pi Zero W has been benchmarked through tests of transferring a file of 200 megabytes via raw TCP between nodes twice, and averaging the values. They are reported in Table $\mathrm{I}$

TABLE I: Signal Strength \& Data Rate Tests between Nodes

\begin{tabular}{|c|c|c|}
\hline Distance & Data Rate & Signal Strength \\
\hline $60 \mathrm{~m}^{\mathrm{i}}$ & $73 \mathrm{mbits} / \mathrm{s}$ & $-50 \mathrm{dBm}$ \\
\hline $70 \mathrm{~m}^{\mathrm{ii}}$ & $27 \mathrm{mbits} / \mathrm{s}$ & $-76 \mathrm{dBm}$ \\
\hline $100 \mathrm{~m}^{\text {iii }}$ & $12 \mathrm{mbits} / \mathrm{s}$ & $-76 \mathrm{dBm}$ \\
\hline $100 \mathrm{~m}^{\mathrm{iv}}$ & $15 \mathrm{mbits} / \mathrm{s}$ & $-80 \mathrm{dBm}$ \\
\hline
\end{tabular}

The bitrate of a 16-bit, stereo WAV file with a sampling rate $F_{s}=44100 \mathrm{~Hz}$ is $1411.2 \mathrm{kbps}$ (CD-quality audio standard [17]) and $320 \mathrm{kbps}$ is the typical bitrate for high-quality lossy MP3. Both of these requirements fall far under the measured

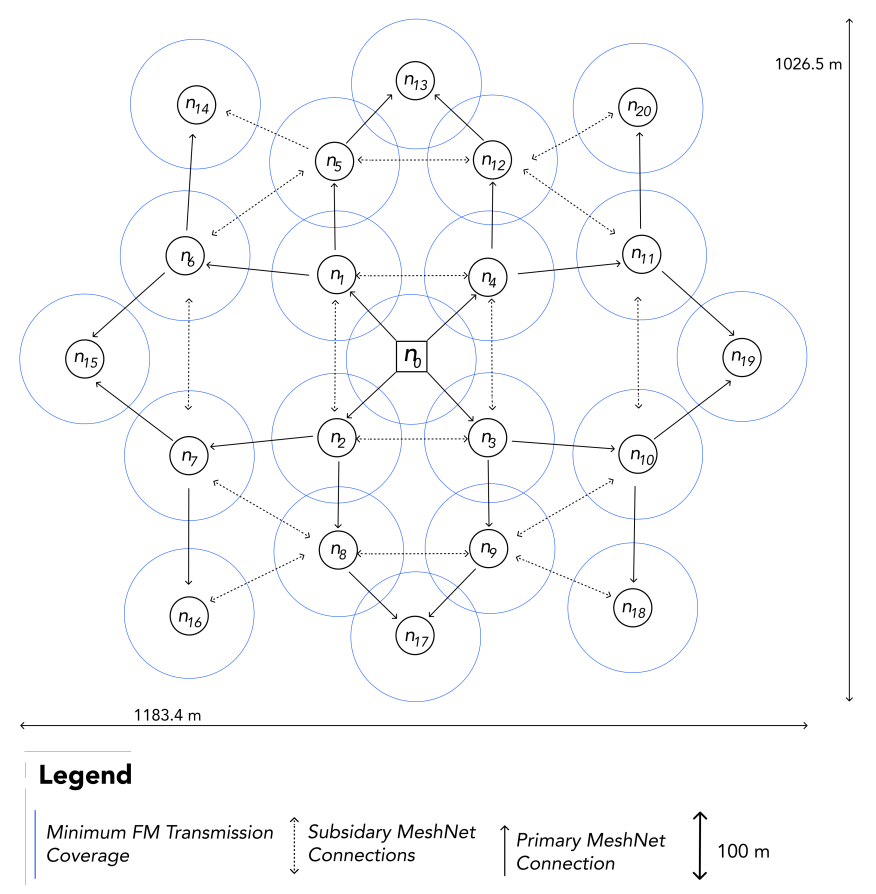

Fig. 11: 20-Node WiFi MeshNet Setup for DAB (to scale)

data transmission rates at a distance of $100 \mathrm{~m}$, so each node caches audio when recordings are broadcast, not requiring a consistent connection throughout the duration of the broadcast.

The cost analysis for a system with 20 nodes was examined in Appendix A, with an estimated cumulative cost of 87,855 INR. In Figure 11 the configuration functions with the assumption of two 0.3 metre concrete walls between each node. A maximum distance of 100 metres between nodes (furthest tested WiFi reception) is taken as an assumption to keep the network dense, with the maximum DAB distance being approximately 60 metres (testing done in Experiment 2). A receiver is expected to be closer than 60 metres away from at least a single node. The default power state of the nodes may be idle, from which they can be set to transmit through instructions via the WiFi Mesh Network. The network covers a rectangular area with a breadth of 1,183 metres, a length of 1,027 metres, and a large coverage area of $1,214,760 \mathrm{~m}^{2}$, although with notable gaps. The network must be configured such, as to ensure equitable access 
to all inhabitants of an area. This can be achieved through trial and error, and developed through further research.

\section{CONCLUSION}

The system performs favourably in testing for scaling and quality. COTS components remain easy to replace and upgrade, providing a scalable option for deploying community radio systems, especially in uneven terrain and radio-sensitive areas due to selective coverage. Additionally, for a broadcast of recorded audio, the system does not require a consistent connection due to caching.

Section III-D analyses a real-world scenario with empirical data for a closer look at a potential deployment with lowcost solar panels on the device itself (see Appendix A). The device will have a low ERP, and may face reduced regulatory difficulties due to the same.

A need has been expressed by different parties, including governments, for innovations in Community Radio transmission [18], and by international organisations such as the UN [10], due to their potential for cultural and social impact. The authors hope that the application of their proposed method will aid in increasing the accessibility and reach of local broadcasts, for both local broadcasters as well as receivers.

\section{APPENDIX A Cost CONSIDERATIONS}

A low-end smartphone has an Indian pricing of approximately [19] 6,500 Indian Rupees (INR), far more expensive than the common feature phone with Indian pricing of 1,000 INR [20].

TABLE II: Cost Analysis for a MeshNet of Transmitters

\begin{tabular}{|l|c|r|} 
Component & Quantity & Cost (INR) \\
\hline Raspberry Pi Zero & 20 & $29600[21]$ \\
WH & 40 & $55[22]$ \\
Female-Male Wire & & $40000[23]$ \\
IFITech Solar Panel $^{\mathrm{v}}$ & 20 & $18000[24]$ \\
PowerBank & 20 & $200[25]$ \\
Microphone & 1 & $\mathbf{8 7 8 5 5}$ \\
\hline Total & 20 nodes & \\
&
\end{tabular}

Table III gives a cost estimate for setup of a network of 20 nodes powered by solar panels, with each device powered by the USB port of a powerbank (PLM13ZM by Xiaomi is used with $10 \mathrm{Ah}$ at the nominal voltage of $3.6 \mathrm{~V}$, containing ideally $133.2 \mathrm{~kJ}$ of energy) with a maximum power draw of $7.2 \mathrm{~W}(1.2 \mathrm{~A} / 6 \mathrm{~V})$. On idle with $\mathrm{WiFi}$ active, the Pi consumes $0.25 \mathrm{~A}[26]$. For the solar panel, the IFITech Portable Solar Charger Panel [23] is used, which can deliver upto 5V, 6W regulated power to charge the powerbank.

For the given setup, with $4.16 \cdot 10^{-2} \mathrm{~m}^{2}$ panel area, and a specified 17\%-20\% energy transformation efficiency [23], and likely immobility, daily solar radiant exposure is considered for energy generation. Considering an example of the cloudy region of Shillong $([27])$, there is an annual daily output of $16.27 \frac{\mathrm{MJ}}{\mathrm{m}^{2} \text {.day }}$, and lower cap of $14.11 \frac{\mathrm{MJ}}{\mathrm{m}^{2} \cdot \text { day }}$, i.e $115.06 \mathrm{~J}$ considering the selected solar panels. Taking an idle load of $0.25 \mathrm{~A}$ for 10 hours, and active load of $1 \mathrm{~A}$ across a $6 \mathrm{~V}$ circuit for 1 hour, the total daily energy usage would approximately be $21 \mathrm{~J}, 1 / 5$ of the estimated generation. Further experiments should help explore further configurations.

\section{ACKNOWLEDGMENT}

The authors would like to thank the Head of Computer Science at their high school, Delhi Public School R.K Puram, Mr. Mukesh Kumar, for providing them with the platform to come together and develop the idea, and particularly like to thank Mr. Pranshu Malik, for aiding them with all his expertise in the technicalities involved and being the project mentor.

The authors would also like to extend their gratitude towards their colleague Mr. Alankar Dutta for his help in understanding audio data, and lastly towards a few colleagues who aided in editing, Mr. Sagnik Anupam, Mr. Aditya Swaminathan, Mr. Aditya Sengupta, Ms. Pearl Dhingra.

\section{REFERENCES}

[1] Ministry of Information and Broadcasting, "Guidelines for Community Radio Support Scheme (CRSS).” [Online]. Available: https://mib.gov.in/sites/default/files/Guideline s_for_financial_assistance_under_CRSS.pdf

[2] S. Estrada and C. Fraser, Community Radio Handbook. UNESCO, 2001.

[3] T. R. Humphrey, "A radio-relay remote-control system for fm broadcast stations," Electrical Engineering, vol. 73, no. 4, pp. 327-330, April 1954.

[4] C. Rey-Moreno, Z. Roro, W. D. Tucker, M. J. Siya, N. J. Bidwell, and J. Simo-Reigadas, "Experiences, challenges and lessons from rolling out a rural WiFi mesh network," in Proceedings of the 3rd ACM Symposium on Computing for Development - ACM DEV '13. ACM Press, 2013.

[5] Government of India, "Schedule VIII," in Constitution of India, 1950.

[6] — - "Census of India 2011," OFFICE OF THE REGISTRAR GENERAL, INDIA 2A, MANSINGH ROAD, NEW DELHI 110011, Tech. Rep., 2011.

[7] Ministry of Information and Broadcasting, "Study on Listenership, Reach, Effectiveness of Community Radio Stations in India," Ministry of Information and Broadcasting, Tech. Rep.

[8] A. Shiundu, "More Must Happen," 2018. [Online]. Available: https://www.dandc.eu/en/article/literacy-rates-h ave-risen-sub-saharan-africa-reality-probably-worse-of ficial-numbers-suggest

[9] S. Elders, "African Languages. An Introduction: Bernd Heine and Derek Nurse, Cambridge: Cambridge University Press.," Lingua, vol. 113, pp. 1255-1265, dec 2003.

[10] I. Madamombe, "Community radio: a voice for the poor," Africa Renewal, 2005. [Online]. Available: https://www.un.org/africarenewal/magazine/july-2005/co mmunity-radio-voice-poor 
[11] L. Manyozo, "Mobilizing rural and community radio in africa," Ecquid Novi: African Journalism Studies, vol. 30, no. 1, pp. 1-23, Jan. 2009.

[12] G. R. Hiertz, D. Denteneer, S. Max, R. Taori, J. Cardona, L. Berlemann, and B. Walke, "IEEE 802.11s: The WLAN Mesh Standard," IEEE Wireless Communications, 2010.

[13] International Telecommunication Union, "FM / TV Regional Frequency Assignment Plans."

[14] Raspberry Pi Foundation, Raspberry Pi Zero W, 2018.

[15] J. Miegl, "PiFmAdv," Jul. 2019. [Online]. Available: https://github.com/miegl/PiFmAdv

[16] J. O. Smith, Spectral Audio Signal Processing.

[17] International Electrotechnical Commission, Compact Disc Digital Audio System, 2nd ed., 1999.

[18] Commonwealth Educational Media Centre for Asia, "Innovations in Community Radio," Ministry of Information and Broadcasting, Government of India, Tech. Rep., 2014.

[19] Amazon.com Inc, "Samsung Galaxy M01 Core." [Online]. Available: https://www.amazon.in/Samsung-Galaxy-Stora ge-Additional-Exchange/dp/B089MTL51J/

[20] -, "Inovu A9i Dual Sim Feature Mobile Phone with 800 mAh Battery." [Online]. Available: https: //www.amazon.in/Inovu-A9i-Feature-Mobile-Battery/dp/ B07MJXDB8R/

[21] Robu.in, "Raspberry Pi Zero Wireless WH (Pre-Soldered Header)." [Online]. Available: https://robu.in/product/rasp berry-pi-zero-wireless-wh-pre-soldered-header/

[22] - "Male to Female Jumper Wires 40 Pcs 10cm." [Online]. Available: https://robu.in/product/male-to-fema le-jumper-wires-40-pcs-10cm/

[23] Amazon.com Inc, "IFITech 6W Portable Solar Charger Panel.” [Online]. Available: https://www.amazon.in/dp/B 07V4FFMRG/

[24] Amazon.com Inc., "Mi Power Bank 3i 10000mAh Dual Output and Input Port." [Online]. Available: https://www.amazon.in/Power-10000mAh-Metallic-Ou tput-Charging/dp/B08HVJCW95/

[25] Robu.in, "Raspberry Pi USB Plug n Play Desktop Microphone." [Online]. Available: https://robu.in/product/ raspberry-pi-usb-plug-and-play-desktop-microphone/

[26] Matt, "Raspberry Pi Power Consumption Data," Raspberry Spy, nov 2018.

[27] Solar Energy Centre of Ministry of New \& Renewable Energy, "Solar Radiation Hand Book," Indian Metrological Department, Tech. Rep., 2008. 